\title{
Noise and Feedback in Online Communication on Sex: A Study of Nigerian's Conversations on Pornography in Nollywood on Social Networks
}

\author{
F. P. C. Endong \\ Department of Theatre and Media Studies, University of Calabar, PMB 1115, CRS, Calabar-Nigeria \\ Email: floribertendong@yahoo.com
}

\begin{abstract}
Social networks constitute a suitable forum for debate, and exchange on any sort of topic especially highly sensitive issues. They offer a fertile platform for debate on thorny societal issues such as politics, sex, culture and religion among others. Given the fact that they favor anonymity, openness and non-accountability for voiced opinion, a good number of Nigerians have found them suitable for, "hot", "aggressive" and very passionate discussions over subjects like sex, sexuality and religious convictions - issues which have remarkably remained somehow taboos in the Nigerian society. This paper investigates the conduct of online debates and opinion formation on sex in the prolific Nigerian motion picture industry (Nollywood). It is based on the content analysis of 516 comments by Nigerians, reacting or debating online (in social networks) on pornography in the Nigerian film industry. The paper seeks to explore and quantify the phenomenon of noise in online communication (conservation and debate) on sex by Nigerians. It equally examines how this noise affects communication flow in online debate on pornography in the Nigerian film industry. It argues that being somewhat considerable, noise in such a communication context, is mainly psychological in nature, due principally to the dominance of conservative beliefs on sex and pornography in the Nigerian society. This conservatism motivates most Nigerians to mainly have preconceived stereotypes, notions and biases on sex and pornography and to adopt judgmental and censuring reactions to most attempts to celebrate pornography. The effect of the psychological noise (as observed in online conversation on pornography) is mainly to orchestrate a change of topic from sex to other sensitive issues as politics and religion or engender insults and counter insults which further negatively affect communication.
\end{abstract}

Index Terms-Social Media, Noise, Feedback, Communication Process, Encoding-Decoding Theory.

\section{INTRODUCTION}

Sex in fact and symbol has always been of continual burning interest to mankind. Indeed, the phenomenon is today recognized as an attractive topic for reflection, debate, communication and exchange. In line with this, scholars like Williams and Miller have remarked that there is an unarguable obsession by the modern man especially the Westerner - with talking about and knowing sex, so as to derive an imaginary pleasure from these two exercises (talking and knowing sex) [1-3]. Talking about and knowing sex - particularly personal sexual desires - is, in this respect, believed to have the potential of permitting man to immensely learn about personal truth, since sex is often construed as the secret [2, 4]. This obsession has been termed by William as "the modern compulsion to speak incessantly about sex" [1]. Having relatively religious and western origins, this compulsion started with the progressive transformation of sex into a discourse. As noted by Wiesner-Hanks, the process (that of making sex a cardinal topic for public discourse) began with the Christian practice of confessing one's sins to a priest, "during which first acts and then thoughts and desires had to be described in language. This practice expanded after the Reformation as Catholics required more extensive and frequent confession and Protestants substituted the personal examination of conscience for oral confession to a priest" [3]. The practice evolved, became more subtle and complex, and finally found its way into modern times. Foucault traces the expansion of the discourse about sex into contemporary times in his statement "[today], we talk more about sex than anything else" [4].

The modern compulsion to talk incessantly about sex has thus given birth to such contentious phenomena and neologisms as pornography, mediated sex, obscenity, and pornotopia/pornophobia. With African communities becoming more and more influenced by Western cultures, pornography and obscenity have progressively found their way into public discourse, and this, not without criticisms. In line with this, mediated sex is a reality in contemporary Nigeria. Almost all media of mass communication (music, film, magazines and the like) have found a way to incorporate discourse on sex and absorb pornography [5-7]. Sex is also fuelling highly virulent debates online (on social networks). However, due to concurrent attitudes and biases towards the phenomenon (sex), discussions in the media about it are always the site of conflicting opinions, sometimes expressed through aggressive an impolite language. The nature of messages, noise and feedback in communication processes involving talking about sex is 
therefore determined by such concurrent attitudes and biases. This paper seeks to examine how social, psychological and semantic forms of noise affect the feedback of Nigerian commenters's reactions to online critiques of Nollywood films, particularly critiques censuring the heavy sexualization of the industry. It specifically aims at examining the degree at which online discussion on sex attracts reactions from Nigerians and how social, psychological and semantic forms of noise subtly determine the nature of feedback in such a complex communication context.

\section{THEORETICAL FRAMEWORK}

This paper is anchored on Gudykunst's Anxiety/Uncertainty Management (AUM) theory and Hall's encoding and decoding theory. The AUM theory defines how human communication is affected by individual communicators' balance of anxiety and uncertainties in specific social situations. According to this theory, intercultural communication has chances to be effective when there is a remarkable reduction of anxiety or uncertainty. This indicates that AUM theory is associated with Berger and Calabrese's Uncertainty Reduction Theory (URT), introduced in 1974. In the context of AUM theory, anxiety is an apprehension based on fear of negative consequences. The phenomenon is prevalent in most intergroup relations and interactions due to the fact that there is an added fear of appearing prejudiced when dealing with an outgroup. The AUM Theory holds that effective communication is conditioned by the interlocutor's ability to manage and keep anxiety/uncertainty between minimum and maximum thresholds. Once one reaches his/her highest level of anxiety, virtually all his/her attention is focused on the source of this anxiety rather than on achieving effective communication. Conversely, optimum level of anxiety will engender trust and self confidence that is needed for effective communication. The idea of managing levels of anxiety and uncertainty could be compared to managing stress and distress to ultimately arrive at optimum performance in a communication context.

The Anxiety/Uncertainty theory therefore focuses principally on interpersonal and intergroup levels of communication. The first level borders on the way in which the message is exchanged by encoder/sender and decoder/receiver in one-on-one communication context or when they intimate relationships and social networks. The second (intergroup communication level) occurs in contexts of collective exchange of messages by human, for instance as a strategy to perpetrate social identities or collective self-esteem. By examining predictions of behavior, AUM discriminates between interpersonal and intergroup communications. Behaviors that are explained with cultural or sociological norms are classified as intergroup communication meanwhile behaviors that are best explained with psychological norms are considered interpersonal communication. Based on this differentiation, the theory holds that when human behavior is guided by personal or human factors, interpersonal behaviors tend to occur. On the other hand, when it is guided by social factors, the opposite is true.

The AUM theory has numerous assumptions. The following highlights are selected postulations that are relevant to this study:

(i) The basic processes of communication do not differ across cultures; only the methods of interpretation vary.

(ii) When humans are mindful of cultural and societal differences they have greater control over their communication behaviors.

(iii) Strangers generally trigger both interpersonal and intergroup anxiety. It is important to note here that the perspective considered by the AUM Theory is that of the stranger immersed in an ingroup.

(iv) When uncertainty is moderate - that is when it falls between an individual's minimum and maximum acceptable levels - effective communication is bound to take place. The maximum threshold is regarded as the amount of uncertainty that a human can possess and still comfortably predict the behavior of a stranger. On the other hand, uncertainty above the minimum threshold keeps us from getting bored with the stranger and hence constraining communication.

This paper equally hinges on Hall's encoding/decoding theory. The encoding and decoding theory is one of the multiple theories often envisaged in reception analysis, (an approach which seeks to associate the attribution and construction of media text's meaning with the receiver). The encoding/decoding theory emphasizes the different stages mass media messages pass through in the process of transmission from source (origins) to reception and interpretation. Being somehow antithetical to claims in the structuralism and semiology that the meaning of media text is embedded in itself, Hall's theory stipulates that communicators are generally kind of "spin agents" who deliberately encode their messages for ideological objectives, manipulating language and the media they use for such ideological ends. Through such a subtle approach, encoded messages are given a preferred meaning otherwise known as "spin". However, receivers do not automatically accept, decode or interpret communicator's messages as sent. They sometime resist the ideological influences (they detect in the message), by systematically or instinctively applying concurrent and very antithetical readings or interpretations, this depending on individual experience and cultural background. In line with this, the same message may concurrently be perceived by people from different subculture, especially as they apply mutually antithetical reading of the message. Such application of antithetical readings likely leads to a degree of noise and eventually to ineffective communication. 


\section{NOISE AND FEEDBACK IN THE COMMUNICATION PROCESS}

Noise and feedback are two cardinal components of the (mass) communication process. Being inter-related, the two factors greatly determine the quality of communication. Noise can be defined as any form of interference which may affect the delivery of the message in a communication context. This indicates that noise can prevent formulated messages from reaching their destination or from being understood as intended by the sender. As Dominick remarks, "a little noise might pass unnoticed, while too much noise might prevent the message from reaching its destination [...] As noise increases, message fidelity (how closely the message that is sent resemble the message that is received) goes down" [8]. Most communication scholars limit to three, the types of noise to be envisaged in a communication process. These forms of noise include the semantic, mechanical and environmental types. However, noise can be categorized into seven types namely social/cultural, physiological, environmental, semantic, psychological, syntactical and channel/mechanical [9-10]. This paper focuses on three out the lot, namely social, semantic and psychological.

Social noise has to do with interferences (barriers) occurring when the social and cultural backgrounds of the sender and receiver and persons who are included in the process of communication are diametrically divergent. Such interferences are manifested for instance, in multiethnic community when a receiver adopts an unexpected (negative) reaction to a message which, under normal circumstances, was to trigger a positive feedback. Some scholars also refer to this type of noise as cultural noise. In principle, a cultural noise occurs in situations where, due to diametrical differences between the culture or subculture of the audience and that of the sender, message is misunderstood (that is, such a message is understood in [a] way the sender might not have anticipated). Socio-cultural noise is bound to occur in cross-cultural communication contexts and the greater the divergences in culture, the more noise in likely to interfere in the communication. O'Sullivan et al note this scenario in their discussion of the encoding decoding theory thus:

Often, different groups of people (or sub-cultures) will share quite distinct social locations and perceive the world and the messages of the media accordingly. While the general implication is that meaning as decoded does not necessarily (or often) correspond with meaning as encoded (despite the mediation of conventional genres and language systems), the most significant point is that decoding can take a quite different course from that intended. Receiver can read between the lines and even reverse and 'subvert' the intended direction of the message. [11]

Psychological noise otherwise called internal noise refers to the person's (communicator's) partiality, chauvinism, and emotion toward a message or a person (his interlocutor). Psychological noise is therefore the consequence of preconceived notions a communicator brings to the communication process, such as racial stereotypes, reputations, biases, and assumptions. It is also connected with preconceived attitudes and biases towards others, event, situations and topics. Semantic noise on the other hand is associated with the semantics of words. It occurs when concurrent meanings are given to the same word or phrase in a given message or when the order of such words confuses the meaning of the message. This is sometimes encountered in situation where people from certain profession as law, physics, medicine and the like use such complicated jargon that may only confuse receivers and potentially make communication ineffective. Dominick illustrates a similar scenario when she notes that: If you ask a New Yorker for a "soda" and expect to receive something that has ice cream in it, you'll be disappointed. The New Yorker will give you a bottle of what is called "pop" in the Midwest" [8].

Noise is related to feedback in that, the greater the potential for quick (immediate) feedback, the greater the chance to overcome noise. In effect, to reduce psychological noise, the sender and receiver must concentrate on the message and try to control the psychological barriers. In the case of semantic noise, the sender and receiver must consider their socio-cultural differences and concentrate on message positively keeping aside these differences, when ever noises have been recognized. For effective communication, it is always advised to deal promptly with noise. In line with this, relevant feedback is a solution to noise and effective communication. By definition, feedback is the set of responses by the receivers which shape and determine subsequent messages from the sender. Feedback permits the source or sender to evaluate and monitor his or her communication and if necessary, effect changes in the elements of the communication process. Feedback can be positive (when it encourages the communication behavior in progress) or negative (when it attempts to change or terminate the communication). Noise has generally been branded as a negative aspect of communication as it is generally regarded as a serious threat to effective communication. However, contemporary construction have somewhat changed. Aly et al note that:

Today, noise is breaking away from the status of undesirable phenomenon bestowed upon it by traditional communications theory. No longer merely an undesirable element to be eradicated so as to retain the purity of the original signal, noise can be regarded as a more complex and even desired element. When it comes to the terrorist (or any other illegal, harming, and dangerous communication), one may question the instrumentality of creating noise that may reduce the communicator's efficiency and success. Creating and using semantic, psychological, cultural, and physical noises may describe a rich variety of counter measures and organize them in a strategic framework. Thus, 
noise could become a key conceptual and theoretical foundation in the strategy of countering terrorism online. [10]

Based on this premise a good number of government and international organizations have, since the emergence of terrorist groups in the world, designed relevant and formidable strategies involving the use of 'noise' to handicapped terrorist propaganda in the world. The social networks constitute one of the "battle fields" where this form of counter noise has been used to frustrate terrorists and other illegal movement's communication efforts.

\section{FEEDBACK IN SOCIAL NETWORKS}

As very complex forms of online communication, the social networks (facebook, Twitter, google+, YouTube, Myspace, Flickr, and the like) use a wide range of special techniques involving participation, conversation, linkage, collaboration and sharing to facilitate exchange of information between source or encoder(s) and receiver(s). Through these various techniques, feedback is systematically and artfully generated from people on various issues or items. An online post in a Blog or Web site which motivates people to discuss and exchange on a particular issue is a very good illustration of the conversation function of social networks. Through such conversations there are subsequent, rich, and provocative reactions (feedback) by posters and commenters on the issue discussed, as people have the opportunity to comment and respond to one another. Similarly, an online news or feature article that solicits responses from potential viewers or readers and present particular motions to them is a relevant illustration of the participatory function of the social media. Web operation such as Digg even permits people to vote for their favorite online article or image, thereby facilitating participation and generation of feedback by users of social networks.

The theoretical lack - or reduced nature - of accountability for one's voiced opinion, decision or action in online communication context (due mainly to anonymity) often causes a variety of negative feedbacks such as decreased politeness and more uncivil attitudes to be eventual, imminent or recurrent in social networks. A number of studies have successfully established the fact that these factors (anonymity and lack of accountability of users' action in online discussion) cause commenters to adopt aggressive language (for instance racist, religious or tribalistic/ethnocentric languages), especially when challenged with an oppositional ideology [12-17]. These forms of aggressive language are often observed in conversations, over sensitive issues like politics, religion, sex and sexuality. In effect, online discussions on such sensitive topics are mainly characterized by a glaring polarization whereby like-minded commenters connect with those sharing similar views and interests rather than engaging in objective debate and deliberation with those holding antithetical views, information or opinions [1819]. This somehow points to the possibility for social, cultural and psychological noise to very much affect communication process in case of online discussion on such sensitive issues as sex, religion and politics. In tandem with this, Aly, Weinmann and Weimann note that "as a communication medium and vehicle of influence, the Internet is a powerful tool for psychological campaigns" as it is often used by various quarters to launch online campaigns, "in the same way legitimate political campaigns use the Internet". Such psychological campaigns "attempt to attract users and ultimately seduce them by engaging them in a sensory experience, trying to manipulate their needs, suggesting the fulfillment of a goal, and inspiring and guiding the users to make a choice by providing a higher-level motivation" [10].

\section{SOCIAL NETWORKS AS FORUM FOR DISCUSSION ON SEX}

The social networks have offered audiences multiple opportunities of communication and sharing of information. They have become formidable ways of sharing and obtaining information in contemporary Nigeria. As a popular application service on the Internet, they attract millions of users, including Nigerians. The growth of these media is promoted by users who interact with one another, construct relationships, publish or reply posts, and discuss a variety of topics. Nigerians therefore view them today as golden opportunities and forums to discuss a variety of issues bordering on the political, economic and social life of the country. In line with this, the thorny debate on mediated sex, particularly pornography in the Nigerian motion picture industry (Nollywood) has found its way into the social networks.

The social media permit a high deal of anonymity and openness and because of these factors, commenters often regard them as forum par excellence to discuss topics that are often considered as taboos or highly sensitive. Xiong and Liu stress on the appropriateness of these facilities (social networks) for special kind of communication and opinion formation when they concede that:

The process of opinion formation on social media is more complicated than in real society, and information diffuses and evolves more rapidly. For instance, users always discuss issues with others anonymously. They do not know the true names of their neighbors, and they cannot become well acquainted with the personality characteristics of their neighbors. Moreover, users cannot directly see the internal opinions of their neighbors but instead learn about their opinions through the posts they publish. [20]

Social media are therefore usually coupled with such somewhat promising terms as anonymity, networks, interoperability, interactivity, autonomy, and openness which make the discussion of sensitive issues and taboo by users or commenters less "risky". As Ketchum succinctly puts it, the social media "create unprecedented opportunities for the democratization of information and the liberation of stifled and even abject "knowledges"' 
[21]. In tandem with this, Yu-Won contends that the relatively anonymous character of online communication, in particular, is believed by most communication theorists to "free individuals from social expectations and constraints, and thus greatly reduces the threats of social sanctions for their behavior" [22]. Quoting Mutz, he further explains that "when people are anonymous and cannot be personally identified with their judgments, the tendency to conform to others' views is considerably attenuated. As a consequence, individuals have opportunities to express their opinions online in ways not possible in their traditional, face-to-face social environment". In line with this, online Nigerian commenters have had the opportunity to discuss issues as pornography, sex and the like without their identity being revealed. A good number of blogs, Facebook and Twitters pages by film reviewers, producer, actors, newspapers and the like exist in the Internet with strings of opinions generated by Nigerians on the issue. Rich and aggressive reactions are made to posts presenting critiques over various aspects of Nollywood including the injection of sex into films of the industry. Esan gives a clue of the active debate staged online on multiple aspects of the industry as technology of production, production aesthetics and exhibition technique. He observes that "Nollywood audiences are aware of the deficiencies in the industry [...] The love - hate affair with the industry is very evident in the online communities where stars are cut down to size for their poor acting and social misdemeanors. Yet they [Nigerian audiences] continue to patronize these stories about their land - their people and their issues" [23]. Pornography in the Nigerian motion picture industry is one of the major issues feeding debates by Nigerian commenters in the various social media. However, though raising a serious debate in these online spaces, the phenomenon seems not to totally "kill" Nigerian audience's loyalty to the industry.

Online commenters' opinions on the injection of pornography into Nollywood films could be categorized in two antithetical classes namely the pro-porn and the anti-porn positions. The anti-porn opinions are rooted in the prevailing conservatist and traditionalist doctrines or school of thoughts, which either advocate for the upholding of religious purism or the flourishing of core Nigerian values in filmmaking as in the designing of other forms of mass media communication. Conservatism and traditionalism attach a lot of secrecy to sex and sexuality and militate against public discussions of sex and mediated sex, viewing such activities as alarming violations of religious and morality codes as well as indicators of identity crisis. Anti-porn opinions therefore relegate pornography to a permissive western value and a total social dysnomia (especially for Africans). Better still, These anti-porn opinions relegate the phenomenon to a sin against God. In tandem with this, Okanatotor refers to pornography as "a crushing and alluring public health danger to our [Nigerian] society, and society needs protection against it" [24]. Such a school of thought opines that the injection of such an artifact (pornography) into Nigerian films, only negatively affects the aesthetics of the films, especially when such a feature is in no way reasonably connected to the plot of the film. The excessive explicit depiction of sex in Nigerian films is thus commonly equated by Nigerian online commenters to an expression and reproduction by most Nigerian film makers of the capitalist culture - a culture which is imported from the western scene. In effect, it has been argued that sex sells and reproduces western capitalistic culture or logic [2]. It has equally been argued that the injection of pornography in African films is an index of African film producer's inferiority complex [5, 25]. As explained by Uwom, Chioma and Sodeinde, most Nigerian film producers and media practitioners tend to see the foreign scenes as being very superior and so, strive extremely hard to meet the needs of the sophisticated and exposed viewers who have now been tremendously influenced by the western pattern of life, if not glaringly westernized. In the bid to satisfy the audience and meet up with what is arguably perceived as international standards, local film and media producers systematically copy international intuition and production techniques (particularly the ones applied in Hollywood films), thereby westernizing - and accidentally/deliberately over-sexualizing their films [26]. Uwom, Chioma and Sodeinde note that, though this approach helps in improving production quality it somehow also leads to the degradation of core Nigerian cultural values [26].

Advocates of the pornography in Nollywood films, on the other hand, commend westernization and sexualization of Nigerian movies, defining/interpreting such phenomena as marks of advancement and modernization. Pro-porn positions are grounded on the rejection on conservatism and traditionalism which are progressively perceived as not being compatible with the dynamics of modernization. Ojukwu and Ezenandu mention a number of conceptions of the modernization which are in line with such positions. They note that:

Modernity or modernization expresses that tradition is outdated and antiquated and so should be wiped out by the process of development. It denotes the transformation of the disparate groups into an integrated and consolidated polity by eliminating the residual set of values, norms and structures of the 'tribal man', and institutionalizing a new set of modern and progressive values [...] The approach suggests that individuals and groups are expected to develop new identities such as modern behavior and refined attitudes to issues thereby shedding off ethnic identities and relationships that are perceived to be pristine. [27]

Censuring pornography on the ground of religion or African tradition is therefore progressively becoming "out dated". In line with this, a sexual personae is regarded by a good number of liberal Nigerians as a professional actor and acting nudity scene has likewise become as normal as just "doing your job". Some of the proponents of this practice (mediated sex/sexualization of media text) even view the incorporation of pornography 
into Nigerian films as inevitable since it arguably represents a strategic instrument that could help emphasize believability of the films. In effect, sex is part of man's life and as Gasper has once argued, "the faulty premise that sex is evil in itself seems to be a delusion unique [not just] to the western world" but equally to the modern Nigerian society [28].

\section{METHODOLOGY}

This paper is based on a content analysis of over 516 comments posted online as feedbacks to over 24 randomly selected articles by Nigerian film reviewers. These articles were written on pornography in the Nollywood film industry and posted on Facebook, Twitter, Online Newspaper/magazine sites and reviewers' blogs. Some of the articles were directly on films deemed pornographic in nature while others were articulated on sexual personae within selected films of the industry. The articles considered for the study actively sought viewers/readers to contribute opinions on the issues discussed. 12 articles had a positive tone (they were slanted in favor of porn in the movies) while the rest provided an antithetical position. The researcher considered only comments by discussants who clearly identified themselves (through names, photos and content of comments) as Nigerians.

Using individual comments as unit of analysis, the study sought firstly to explore and quantify incidences of noise, and secondly to examine the extent to which these forms of noise affected (flow of) communication, especially subsequent comments. Noise was therefore detected based on glaring digressions or extrapolations (by commenters) from the topics discussed and from the implicit or explicit objectives of the various articles posted online. The study systematically differentiated between psychological, cultural and semantic noise, and sought to see how negative, individual types of noise affected feedback (communication). Three principal negative feedbacks were considered for the study (i) change topic of conversation/activity, (ii) engender insults and counter insults, and (iii) terminate communication. Data was collected through the use of a data sheet. These data was analyzed statistically and presented in tables 1, 2 and 3 below.

\section{RESULTS AND DISCUSSION}

Findings indicate that noise is considerably present in online communication on sex, particularly in the discussions by Nigerians over the issue of pornography in Nigerian film industry. The phenomenon actually covers $43.03 \%$ of communication exchanges on the topic (see table 1 below). As show in this table, noise is more accentuated in replies (comments) on articles that are supportive of heavy sexualization of Nollywood films. Over $56.66 \%$ of Nigerian reactions (comments) on proporn articles were various forms of noise meanwhile only $31.81 \%$ noise was noted in reactions to anti porn articles (those articles that seriously condemned pornography in Nollywood films). This is indicative of the fact that most Nigerians still hold conservative beliefs on sex and pornography and will likely adopt negative attitudes towards any attempt at "celebrating" pornography.

Table 1. Noise in Communication on pornography in Nollywood

\begin{tabular}{|l|c|c|c|c|c|c|}
\hline \multirow{2}{*}{ Comment } & \multicolumn{6}{|c|}{ Types of Articles } \\
\cline { 2 - 7 } & \multicolumn{2}{|c|}{ Pro-porn } & \multicolumn{2}{|c|}{ Anti-porn } & \multicolumn{2}{c|}{ Total } \\
\cline { 2 - 7 } & $\mathrm{n}$ & $\%$ & $\mathrm{n}$ & $\%$ & $\mathrm{n}$ & $\%$ \\
\hline $\begin{array}{l}\text { Normal } \\
\text { Comments }\end{array}$ & 101 & 43.34 & 139 & 68.19 & 294 & 56.97 \\
\hline Noise & 132 & 56.66 & 90 & 31.81 & 222 & 43.03 \\
\hline Total & 233 & 100 & 283 & 100 & 516 & 100 \\
\hline
\end{tabular}

Findings also reveal that much $(67.56 \%)$ of this noise is psychological in nature, (see table 2 below). Psychological noise is more accentuated in Nigerians' reactions to anti porn articles as it amount to $80 \%$ of comments in this particular communication context. This finding is similarly justifiable by the fact that Nigerians generally have a lot of preconceived attitudes, biases assumptions, cultural and racial stereotypes on sex (particularly on pornography) as well as on specific advocates of the phenomenon. These preconceived notions are rooted in conservatism, characterized by traditional beliefs and (Christian) religion. Because of such preconceived ideas, most online Nigerian discussants will prefer either to maintain secrecy on sex (by "lobbying" for the closure of the debate on the subject" or superimpose religious concepts that may not have any relation with the objective or the topic of the dialogue or conversation.

Table 2. Type of Noise

\begin{tabular}{|l|c|c|c|c|c|c|}
\hline \multirow{2}{*}{ Type of Noise } & \multicolumn{6}{|c|}{ Types of Articles } \\
\cline { 2 - 7 } & \multicolumn{2}{|c|}{ Pro-porn } & \multicolumn{2}{|c|}{ Anti-porn } & \multicolumn{2}{c|}{ Total } \\
\cline { 2 - 7 } & $\mathrm{n}$ & $\%$ & $\mathrm{n}$ & $\%$ & $\mathrm{n}$ & $\%$ \\
\hline Psychological & 78 & 59.09 & 72 & 80 & 150 & 67.56 \\
\hline Cultural & 18 & 13.63 & 12 & 13.33 & 30 & 13.53 \\
\hline Semantic & 35 & 27.28 & 06 & 6.67 & 42 & 18.91 \\
\hline Total & 123 & 100 & 90 & 100 & 222 & 100 \\
\hline
\end{tabular}

Another important finding here is the fact that cultural noise was less important in the communication process. This is due to the fact that, being all Nigerians, most film reviewers and commenters showed a relatively fair degree of sensitivity to cultural differences in their articles or contributions. Knowing they were communicating or debating principally with fellow Nigerians, the reviewers mostly showed (implicitly or explicitly) that they were mindful of the predominance of the conservative philosophy on pornography and sex among Nigerians. A good number of commenters did same. However, some reviewers and commenters manifested western views about pornography (thereby proffering supportive positions on the phenomenon) and were therefore "treated" by fellow online discussants as cloned westerners. Attitudes towards such "cloned" westerners and their contributions were mostly negative and very aggressive. In some of the reactions commenters 
referred to such cloned westerners as "infidels", "westernized fools", "agents from hell" and the like. Such pejorative appreciations often provoked counter insults which caused serious digressions.

Findings also reveal that the various forms of noise had as dominant effect a change of the topic of discussion or activity. In effect, over $64.86 \%$ of the noise observed in reactions to pro and anti articles coupled, sought to push discussants to change topic of discussion from pornographic content of Nigerian films to other burning issues such as politics and religion. This trend was observed in comments that subtly or glaring extrapolated from the issue discussed to install or encourage diametrically different debates as well in discussants' reactions which simply used the opportunity for advertising their authors' sexual prowess or their "sugar mammy" and "sugar dady" 1 services. Desire to change topic of discussion is more accentuated in comments reacting to pro porn articles. This again confirms the dominant conservative tendency among Nigerians when it comes to supporting or rejecting mediated sex.

Table 3. Effects of Noise

\begin{tabular}{|l|c|c|c|c|c|c|}
\hline \multirow{2}{*}{ Type of Noise } & \multicolumn{6}{|c|}{ Types of Articles } \\
\cline { 2 - 7 } & \multicolumn{2}{|c|}{ Pro-porn } & \multicolumn{2}{|c|}{ Anti-porn } & \multicolumn{2}{c|}{ Total } \\
\cline { 2 - 7 } & $\mathrm{n}$ & $\%$ & $\mathrm{n}$ & $\%$ & $\mathrm{n}$ & $\%$ \\
\hline $\begin{array}{l}\text { Change Topic } \\
\text { or Activity }\end{array}$ & 96 & 72.72 & 48 & 53.33 & 144 & 64.86 \\
\hline $\begin{array}{l}\text { Engender } \\
\text { (counter) insults }\end{array}$ & 30 & 22.22 & 18 & 20.01 & 48 & 21.62 \\
\hline $\begin{array}{l}\text { Terminate } \\
\text { Conversation }\end{array}$ & 06 & 4.56 & 24 & 26.66 & 30 & 13.52 \\
\hline Total & 132 & 100 & 90 & 100 & 222 & 100 \\
\hline
\end{tabular}

Given the sensitive nature of the topic discussed, a good number of commenters caused noise by engendering insults or counter insults. This is shown in the fact that the noise registered in reactions to both types of articles amount to over $21.62 \%$. A relatively small size of the noise sought to terminate communication. This was illustrated by comments that call on reviewers or discussants to stop arguing on pornography as any debate on the issue (according to such a school of thought) is just out of place.

\section{CONCLUSION}

The social networks offer ample opportunities to Nigerians of various ages to passionately debate and discuss over sensitive issues as sex, religion and politics. In line with this, numerous debates and more or less

1 "Sugar Mammy" and "Sugar Dady" are terms commonly used in Nigeria to refer to relatively wealthy and old women and men who are in search for, and sometimes successfully seduced young sexual partners for purely sexual adventures. Sugar mammies and dadies most often preferred partners with serious sexual prowess. Due to extreme poverty, a good number of young Nigerians solicit sexual adventures with such old sexual partners for lucrative purposive. The social networks often offer them a chance to "market" themselves. aggressive discussion have been staged online by Nigerian social network users on sexuality and mediated sex, and particularly on the injection of sex in the prolific Nigerian motion picture industry.

This paper has sought to explore and quantify the phenomenon of noise in online communication (conservation and debate) on sex by Nigerians. It equally examined how this noise affects communication flow in online debate on pornography in the Nigerian film industry. It argued that being somewhat considerable, noise is mainly psychological in nature, due principally to the dominance of conservative beliefs on sex and pornography in the Nigerian society. This conservatism is manifested or characterized by arguments which are implicitly or explicitly based on Christianity and Islam (religious beliefs) and Nigerian traditional values. It pushed most Nigerians to mainly have preconceived stereotypes, notions and biases on sex and pornography and to adopt judgmental and censuring reactions to most attempts to celebrate pornography. The effect of the psychological noise (as observed in online conversation on pornography) is mainly to orchestrate a change of topic from sex to other sensitive issues as politics and religion.

\section{REFERENCES}

[1] L. Williams, Hard Core: Power, Pleasure, and The "Frenzy of the Visible". Berkeley: University of California Press, 1999.

[2] J. Miller, "Kink Unbound (?): Pursuing Pleasure and profit in Pornography" in Schuyikill, Graduate Journal, 34(2), pp. 20-34, 2012.

[3] M. E. Wiesner-Hanks, Christianity and Sexuality in the Early Modern World. London: Routledge, 2012.

[4] M. Foucault, The History of Sexuality I: An Introduction, New York: Random House, 1999.

[5] F. P. Endong, and E.E. Obonganwan, "Sex in Christian Movies: a Study of Roger Young's the Bible: Joseph and Mel Gibson's the Passion of the Christ". IJCMS: International Journal of Communication and Media Sciences, 2(2), 11-21, 2015.

[6] A. Salawu, Sex in Yoruba News Papers" In Salawu (Ed) Indigenous Language Media in Africa. Lagos: Centre for Black and African Arts and Civilisation (CBAAC), 2006, pp.42-63.

[7] A. Ojo, Religion and Sexuality: Individuality, Choice and Sexual Rights in Nigerian Christianity. Lagos: Africa Regional Sexuality Resource Center, 2005.

[8] J. R. Dominick, The Dynamics of Mass Communication (11 $1^{\text {th }}$ Edition). New York: McGrraw Hill, 2011.

[9] C. Nwamuo, "The Nigerian Female Journalist and Communication Faulters". WAACLAS: West African Association for Commonwealth Literature and Language Studies, 3(2), 20-34, 2010.

[10] E. Aly, D. Weimann-Sarks, and G. Weimann, "Making 'Noise': An Analysis of the Say No to Terrorism Online Campaign". Perspective on Terrorism, 8(5), pp.182-199, 2014.

[11] T. O'Sullivan, Key Concepts in Mass Communication. London: Methuen \&Co, Ltd, 1993.

[12] P. Shachaf, and N. Hara, "Beyond Vandalism: Wikipedia Trolls". Journal of Information Science, 36(3), pp. 357370, 2010.

[13] S. Herring, et al. "Searching for Safety Online. Managing 
'Trolling' in Feminist Forums". The Information Society, 18(5), pp. 371-384, 2002.

[14] A. Chadwick, Internet Politics: States, Citizens and New Communication Technologies. Oxford: Oxford University Press, 2006.

[15] T. Huffaker, and S. L. Calvert, "Yes but ... Positive Politeness in Conversation Arguments". Journal of Language and social Psychology, 16(2), pp. 222-239, 1997.

[16] M. Rodino, "Breaking out Binaries: Re-conceptualizing Gender and its Relationship to Language in ComputerMediated Communication". Journal of ComputerMediated Communication, 3(3), pp.17-25, 1997.

[17] M. J. Kushing, and K. Kitchener, "Getting Political on Social Network Sites: Exploring Online Political Discourse on Facebook". First Monday, 14(11), pp.108131, 2009.

[18] N. J. Stroud, "Polarization and Patisan Selective Exposure". Journal of Communication, 60(3), pp.556-576, 2010.

[19] M. Vonover, et al Political Polarization on Twitter in ICWSM, [Online] Available at http://www.aaai.org/ocs/index.ph/ICWSM/ICWSM11/pap er/download2/847/3275. Accessed on the 25/5/2015.

[20] F. Xiong, and Y. Liu, "Opinion Formation on Social Media: An Empirical Approach". Chao: An Interdisciplinary Journal of Nonlinear Sciences, 24(1), pp.193-215, 2014.

[21] E. K. Ketchum, "Gendered Uprisings: Desire, Revolution and the Internet's 'Unintended Consequences"'. Transformation, 23, pp. 121-145, 2013.

[22] O. Yu-Won, "Willingness to Speak out: Comparison Between Online Versus Offline Communication". Korean Journal of Journalism and Mass Communication Studies, 50(3), pp.175-205, 2014.

[23] O. Esan, "Appreciating Nollywood: Audiences and Nigerian Films". Particip@tions, 5(1),pp.68-75, 2008.

[24] O. Okanatotor, "Pornography: Every Young Man's Battle!". Paulines for Life, 4(1), pp. 6-7, 2006.

[25] V. Akande, "If sex is it, let Ghanaian Movies Rule!", The Nation, Saturday January 16. pp. 21-36, 2010.

[26] Unwom, Oguchi et al., Audience Perception of Sexual Contents in Nigerian Movies. New Media and Mass communication, 19, 16-25, 2013.

[27] C. Ojukwu and P. E. Ezenandu. "A Paradigm Shift From Tradition to Modernity in Nollywood's Projection of African Narratives". Global Journal of Human Social Sciences, 12(5), 21-26, 2012.

[28] L. Gasper, Vital Social problems. Berkeley: Mr. Gutcham Publishing Corporation, 1968.
Service (CODEV) as a Public Relation Officer from 2007-2014. $\mathrm{He}$ is author of over 25 journal articles including: "Sex in Christian Movie: A Study of Roger Young's The Bible and Mel Gibson's The Passion of the Christ", International Journal of Communication and Media Sciences 2(2), 11-21, 2015; "The Effectiveness of Advertising/Marketing Messages by Nigerian Manufacturers", Journal of Print and Media Technology Research, 24(2), 129-139; "Westernization as a Threat to the Indigenization of Media Broadcast in Nigeria". Journal of Media and Communication Science 6(8), 148-159. His present research interests include the effects of Nollywood on the Nigerian brand.

Mr. Endong is assessor with over three international journal in media, linguistic and communication sciences.

How to cite this paper: F. P. C. Endong,"Noise and Feedback in Online Communication on Sex: A Study of Nigerian's Conversations on Pornography in Nollywood on Social Networks", International Journal of Information Technology and Computer Science(IJITCS), vol.7, no.12, pp.59-66, 2015. DOI: $10.5815 /$ ijitcs.2015.12.07

\section{Author's Profile}

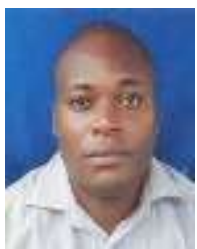

F. P. C. Endong was born in Bouraka, Cameroon, in 1983. He attended the universities of Buea (Cameroon) and Calabar (Nigeria) where he respectively obtained a Bsc in Journalism and Mass Communication in 2008 and a M.A. degree in Media Art Studies (in 2013). He is presently a $\mathrm{PhD}$ scholar at the Department of Theatre and Media Studies, University of Calabar, Nigeria.

He has worked with the Christian Broadcasting Service Buea as a Voluntary External Broadcaster (VEB) from 2007-2010. $\mathrm{He}$ has equally worked with Conservation and Development 